\title{
VALORACIÓN URODINÁMICA EN EL CÁNCER DE PRÓSTATA CON INCONTINENCIA
}

Santiago Méndez Rubio', Jesús Salinas Casado, Miguel Virseda Chamorro, Leandro Chiarelli y Ángel Silmi Moyano.

Servicio de Urología. Hospital Clínico de San Carlos. Universidad Complutense de Madrid. 'Servicio de Urología. Hospital Sanitas La Moraleja. Madrid. España.

Resumen.- OBJETIVO: Puesta al día sobre la valoración urodinámica en los pacientes con cáncer de próstata que presentan incontinencia.

MÉTODOS: Revisión de los estudios urodinámicos en pacientes con cáncer de próstata que padecen incontinencia, sea cual sea el tratamiento efectuado.

RESULTADOS: La información más numerosa sobre estudios urodinámicos en los pacientes con cáncer de próstata e incontinencia se refiere a la incontinencia post-prostatectomía. Son mucho más escasos los trabajos referidos a pacientes radiados y postratamiento farmacológico.

CONCLUSIÓN: El estudio urodinámico en estos pacientes puede ayudar a aclarar los mecanismos etio-

\section{CORRESPONDENCIA}

Santiago Méndez Rubio

Servicio Urología

Hospital La Moraleja

Avda. Pi y Margall, 81

28050 Madrid. (España)

santiago.mendez@telefonica.net

jsalinascasado@yahoo.es patogénicos de la incontinencia, a tratar de predecir factores de riesgo pre tratamiento y ayudar a la toma de decisión terapeútica. Son necesarios más estudios, sobre todo en pacientes sometidos a radioterapia, para evaluar con mayor profundidad los mecanismos implicados en las alteraciones del tracto urinario inferior que se producen en estos pacientes.

Palabras clave: Urodinámica. Cáncer de próstata. Incontinencia urinaria.

Summary.- OBJECTIVES: Update on the urodynamic evaluation in patients with prostate cancer who have incontinence.

METHODS: To review the urodynamic studies in prostate cancer patients who suffer from incontinence, irrespective of the treatment carried out.

RESULTS: The largest amount of information on urodynamic studies in patients with prostate cancer and incontinence was found in those with post-prostatectomy incontinence. There is much more limited work on irradiated patients, and after medical treatment.

CONCLUSION: The urodynamic study in these patients may help clarify the etiopathogenic mechanisms of incontinence, try to predict risk factors before treatment and help the therapeutic decision making. Further studies are needed, especially in patients undergoing radiotherapy, to further assess the mechanisms involved in lower urinary tract abnormalities that occur in these patients.

Keywords: Urodynamics. Prostate cancer. Urinary incontinence. 


\section{INTRODUCCIÓN}

La epidemiología de la incontinencia urinaria en los pacientes con cáncer de próstata es difícil de conocer. Los motivos son varios: La definición de incontinencia utilizada no está unificada, la valoración del periodo pre-tratamiento es escasa, en el postratamiento existe dispersión de resultados respecto a las modalidades terapéuticas empleadas, radicales o paliativas, conservadoras o quirúrgicas, los diferentes modos de radioterapia, además de la imbricación y solapamiento en muchos de los pacientes con la $\mathrm{Hi}$ pertrofia Benigna de Próstata (HBP). Por otro lado, la incidencia de incontinencia en pacientes ancianos no es despreciable, y en ellos puede concurrir también el cáncer de próstata.

La disfunción miccional, en pacientes de edad avanzada no es una entidad uniforme. Las enfermedades del sistema nervioso central, los desequilibrios metabólicos, el envejecimiento del detrusor y la obstrucción vesical o el deterioro del esfínter pueden dar lugar a problemas como la retención urinaria y / o incontinencia urinaria como nos lo recuerda Melchior H (1995) (1).

Según Goode PS et al. 2008 (2) en un estudio con 496 hombres de 65 a 106 años de edad (edad media 75), encontraron una prevalencia de incontinencia del $27 \%$ de los hombres, definiendo la incontinencia como cualquier grado de incontinencia que se produzca al menos una vez al mes en los últimos 6 meses.

Markland AD 2008 (3), por su parte, aunque encuentra diferencias entre la incontinencia en mujeres según su raza (mayor en la blanca), no encuentra diferencias en los hombres, situando una prevalencia del 22,2 \%. Se correlacionó la incontinencia con problemas prostáticos y estados depresivos.

La variabilidad entre los datos de incontinencia es grande. Como señala Thom D 1998 (4) del 2 al $55 \%$. Realizando un metanálisis de 21 estudios, encontraron, para los hombres de mayor edad, la prevalencia de incontinencia se estima en 11 a 34\%, y 2 a un $11 \%$ informaron incontinencia diaria. Entre los de mediana edad y los adultos más jóvenes, del 3 al $5 \%$ para hombres. Hubo una tendencia a que los estudios que utilizan las entrevistas en persona para informar obtuvieran mayores prevalencias.

Irwin DE, et al. en 2006 (5) con una gran muestra de cerca de 20000 personas, encuentra algún tipo de incontinencia en el 5,4\% de los hombres, con una variación según la edad que va de de un $2,4 \%$ en varones de 39 años o menos, un $5,2 \%$ de 40 a 59 años y hasta un 10,4\% en los mayores de 60 años.

La edad de presentación del cáncer de próstata va aumentando a partir de los 50 años. La enfermedad clínicamente poco significativa suele presentarse, como señala Dall'era MA et al. (6) (2009), en pacientes por debajo de los 60 años. Además, como hemos visto, la incontinencia entre los varones de edad avanzada no es un hecho aislado. Por lo tanto, el efectuar la recogida de datos sobre la incontinencia previa a cualquier tratamiento para evaluar la repercusión de éste sobre la continencia urinaria es fundamental. Lamentablemente esto no suele ocurrir con frecuencia.

En otro metanálisis realizado por Hampel C et al. (7) que incluye 48 estudios epidemiológicos entre los años 1954 y 1995, encuentran incontinencia en varones de un 4,6 a un $24 \%$ con una media de $14,5 \%$.

En el estudio EPIC de 2006 (5), realizado mediante encuesta telefónica asistida por ordenador a 58.139 personas de cinco países, de los que se obtuvo repuesta en 19.165 casos, encuentran que un $10.8 \%$ de los hombres presentan síntomas sugerentes de vejiga hiperactiva, de los que un 28,7 presentan incontinencia urinaria y $(41,6 \%$ se debe sólo a urgencia incontinencia aislada, $29 \%$ a otras incontinencias, $19,4 \%$ debido a incontinencias mixtas y un $20 \%$ sólo atribuible a incontinencia de stress).

Pero existe otro factor añadido, que es la imbricación HBP y cáncer de próstata, por lo que los síntomas propios de la HBP pueden ser los predominantes.

Con respecto a los varones con incontinencia urinaria y HPB, encontramos en el estudio de Han $E$ et al. 2007 (8) que la prevalencia en varones de 65 años o más la sitúan entre el 11 y el $35 \%$. Un total de 411.658 hombres con HBP se identificaron a partir de 12.298.027 hombres (3,3\%). La incidencia global de la incontinencia en la HBP de esta muestra fue 1835/100,000/año, y la prevalencia fue $2713 / 100,000$ hombres. Encontraron que el 2,7\% de los hombres con HBP padecían incontinencia. El $57,8 \%$ tenían 65 o más años.

Como vemos, el panorama es complejo. Estamos ante una población entre la que, independientemente de padecer cáncer de próstata o no, presenta con una frecuencia no despreciable incontinencia. La ausencia de estudios urodinámicos entre la población presuntamente sana es evidente. No 


\section{TABLA I. ESTUDIOS URODINÁMICOS NORMALES VS PATOLÓGICOS DEL CÁNCER DE PRÓSTATA NO TRATADO.}

\begin{tabular}{|c|c|c|c|}
\hline Autor & № pacientes & $\begin{array}{c}\text { Estudios urodinámicos } \\
\text { normales }\end{array}$ & $\begin{array}{c}\text { Estudios urodinámicos } \\
\text { anormales }\end{array}$ \\
\hline Aboseif (1994) & 92 & $69,6 \%$ & $30,4 \%$ \\
\hline Hammerer (1997) & 82 & $83 \%$ & $17 \%$ \\
\hline Castille (2003) & 229 & $39,7 \%$ & $60,3 \%$ \\
\hline Majaros(2006) & 63 & $58,7 \%$ & $41,3 \%$ \\
\hline
\end{tabular}

disponemos de estudios poblacionales con estudios urodinámicos que aclaren toda esta situación.

El asunto se complica aún más si tenemos en cuenta la incontinencia debida, bien a cirugía por patología benigna de la próstata o a su evolución.

En un estudio realizado por Nhera $A$ et al. (2009) (9) sobre 2447 hombres de 49 a 79 años de edad, mediante cuestionarios bienales hasta 2007, 226 es decir un $9 \%$ desarrollaron cáncer de próstata durante el seguimiento. De ellos, 143 (64\%) decían no tener incontinencia antes del diagnóstico de cáncer de próstata. De los que no presentaban incontinencia, $60(42 \%)$ presentaron incontinencia un año después del diagnóstico de cáncer. En el último seguimiento después del diagnóstico, un $36 \%$ tenían incontinencia. La relación con el tratamiento quirúrgico ofrecía una odds ratio de 2,5.
Valoración urodinámica de los pacientes con Cáncer de próstata no tratado (Tablas I, II y III).

Siguiendo el esquema propuesto por Tan T L 2003 (10) para la aproximación a la incontinencia en personas mayores deberíamos:

- Identificar y revertir la incontinencia transitoria

- Excluir retención urinaria

- Evaluar la necesidad de pruebas diagnósticas (RNM, urodinámica, etc.)

El estudio urodinámico debe constar de una flujometría libre con medida del flujo miccional máximo $(\mathrm{ml} / \mathrm{s})$, volumen miccional (en $\mathrm{ml}$ ) y residuo postmiccional (en proporción respecto al volumen miccional, una cistomanometría con medida de la capacidad vesical (en $\mathrm{cm} 3$ ), y máxima presión del detrusor en esta fase (en $\mathrm{cmH} 2 \mathrm{O}$ ) y un estudio

TABLA II. HALLAZGOS URODINÁMICOS DE PACIENTES CON CÁNCER ANTES DEL TRATAMIENTO.

\begin{tabular}{|c|c|c|c|c|c|}
\hline Autor & № pacientes & $\begin{array}{c}\text { Hiperactividad } \\
\text { detrusor }\end{array}$ & $\begin{array}{c}\text { Acomodación } \\
\text { disminuida }\end{array}$ & $\begin{array}{c}\text { Afectación } \\
\text { contractilidad }\end{array}$ & Obstrucción \\
\hline Aboseif (1994) & 92 & $14,6 \%$ & - & - & - \\
\hline Golom J (1999) & 20 & $60 \%$ & - & - & - \\
\hline Castille (2003) & 229 & $21 \%$ & 1,7 & $8.3 \%$ & $24 \%$ \\
\hline Gianantoni (2008) & 49 & $55,1 \%$ & $20.4 \%$ & $42,8 \%$ & $57,1 \%$ \\
\hline Majaros (2006) & 63 & $23,8 \%$ & $1,6 \%$ & $3,2 \%$ & $19 \%$ \\
\hline
\end{tabular}


TABLA III. PARÁMETROS DE PERFIL URETRAL PRE Y POST-PROSTATECTOMÍA RADICAL.

\begin{tabular}{|c|c|c|c|c|c|c|c|}
\hline \multirow[t]{3}{*}{ Autor } & \multirow[t]{3}{*}{$\mathrm{N} \cong$ pacientes } & \multicolumn{3}{|c|}{ Longitud uretral Funcional mm } & \multicolumn{3}{|c|}{$\begin{array}{c}\text { Pmxa de cierre uretral en reposo } \\
\text { cmH2O }\end{array}$} \\
\hline & & \multirow[t]{2}{*}{ Preoper } & \multicolumn{2}{|c|}{ postoper } & \multirow[t]{2}{*}{ Preoper } & \multicolumn{2}{|c|}{ postoper } \\
\hline & & & Contin & Incont. & & Contin & Incont. \\
\hline Rudy (1984) & 17 & 43 & \multicolumn{2}{|c|}{16} & - & - & - \\
\hline Salinas (1998) & 25 & 40 & 42 & 35 & 64,7 & 67.25 & 59.87 \\
\hline Hammerer (1997) & 82 & 61 & 27.6 & 20.5 & 89,6 & 68.1 & 53.1 \\
\hline John (2000) & 34 & 50 & \multicolumn{2}{|c|}{24} & 49 & 35 & 11 \\
\hline Presti (1990) & 24 & - & 36 & 21 & - & 74 & 39 \\
\hline
\end{tabular}

presión/flujo con determinación del flujo miccional máximo $(\mathrm{ml} / \mathrm{s})$ y máxima presión del detrusor (en $\mathrm{cm}$ $\mathrm{H} 2 \mathrm{O}$ ). A partir de estos valores, se determina el estado funcional del tracto urinario inferior durante esta fase, utilizando el nomograma propuesto por la ICS y Blaivas (11) (1986). También se puede calcular el número de Abrams \& Griffiths, la resistencia cuadrática y la potencia a flujo máximo (Salinas et al., 2000) (12) (Figuras 1A, 1B Y 1C).

No disponemos de datos en nuestro entorno para evaluar la incidencia y prevalencia de la incontinencia en los pacientes de próstata no tratados.

Rudy DC (13) et al. en 1984 sobre 17 pacientes, encontraron una longitud uretral funcional de $4,3 \mathrm{~cm}$, que se redujo a 1,6 en el postoperatorio. El $19 \%$ de los pacientes presentaban incontinencia clínica o urodinámicamente demostrable en el preoperatorio.

Hellström P et al. (14) de 1989 que estudia a 19 pacientes que iban a ser sometidos a prostatectomía radical, encuentra dos datos en el postoperatorio respecto al preoperatorio que le hacen sospechar una descentralización: un descenso significativo de la acomodación vesical y un incremento de la presión intravesical e intraabdominal durante el flujo máximo. También una presión uretral de cierre baja en dos pacientes totalmente incontinentes.

Aboseif et al. (15) 1994 en su estudio sobre 92 varones, 64 tenían un estudio preoperatorio urodinámico normal y 28 datos patológicos. De estos últimos 12 tenían hiperactividad del detrusor, 9 debi-

\section{ESTUDIO URODINÁMICO COMPLETO}

PACIENTE: $\quad$ EDAD: SEXO:

PROCEDENCIA: ENTIDAD:

HISTORIA CLINICA RESUMIDA:

\section{EXPLORACIÓN FÍSICA NEUROLÓGICA:}

Sensibilidad perineal:

Tono anal:

Reflejo bulbocavernoso:

Control voluntario del esfínter anal:

Próstata:

Vagina:

Otros:

$\begin{array}{ll}\text { FLUJOMETRÍA LIBRE: } & \text { sí } \square \text { NO } \square \\ \text { Volumen de orina } & \mathrm{ml} . \\ \text { Residuo postmiccional } & \mathrm{ml} . \\ \text { Flujo máximo } & \mathrm{ml} \text {. Desv. Estándar Percentil } \\ \text { Flujo medio } & \mathrm{ml} . \quad \text { Desv. Estándar Percentil } \\ \text { Tiempo de micción } & \mathrm{sg} . \\ \text { Tiempo de flujo } & \mathrm{sg} . \\ \text { Morfología: } & \end{array}$

Especificar actividad EMG perineal durante el flujo miccional:

FIGURA 1A. Protocolo del estudio urodinámico completo. Historia clínica, exploración física y flujometría. 
lidad del mecanismo esfinteriano y 7 hiperactividad del esfínter y del detrusor.

Hammerer et al. (16) en 1997, con 82 pacientes, encontraron diferencias estadísticamente significativas entre el pre y el postoperatorio en la presión máxima de cierre uretral. La longitud media funcional disminuye de $61 \mathrm{~mm}$ a $25,9 \mathrm{~mm}$ y la máxima presión de cierre de 89.6 a $65.2 \mathrm{~cm}$. H2O. La capacidad vesical pasa de $338,7 \mathrm{ml}$ a $278,8 \mathrm{ml}$. Se encontró hiperactividad del detrusor en 17 y $41 \%$ de los casos antes e inmediatamente después de la prostatectomía respectivamente. 14 tenían un incremento de presión vesical de más de $15 \mathrm{~cm} \mathrm{H} 2 \mathrm{O}$ durante la cistomanometría de provocación aunque clínicamente silente.

Kleinhans et al. (17) en 1999 encuentra una hiperactividad del detrusor en un $31,8 \%$ de los 66

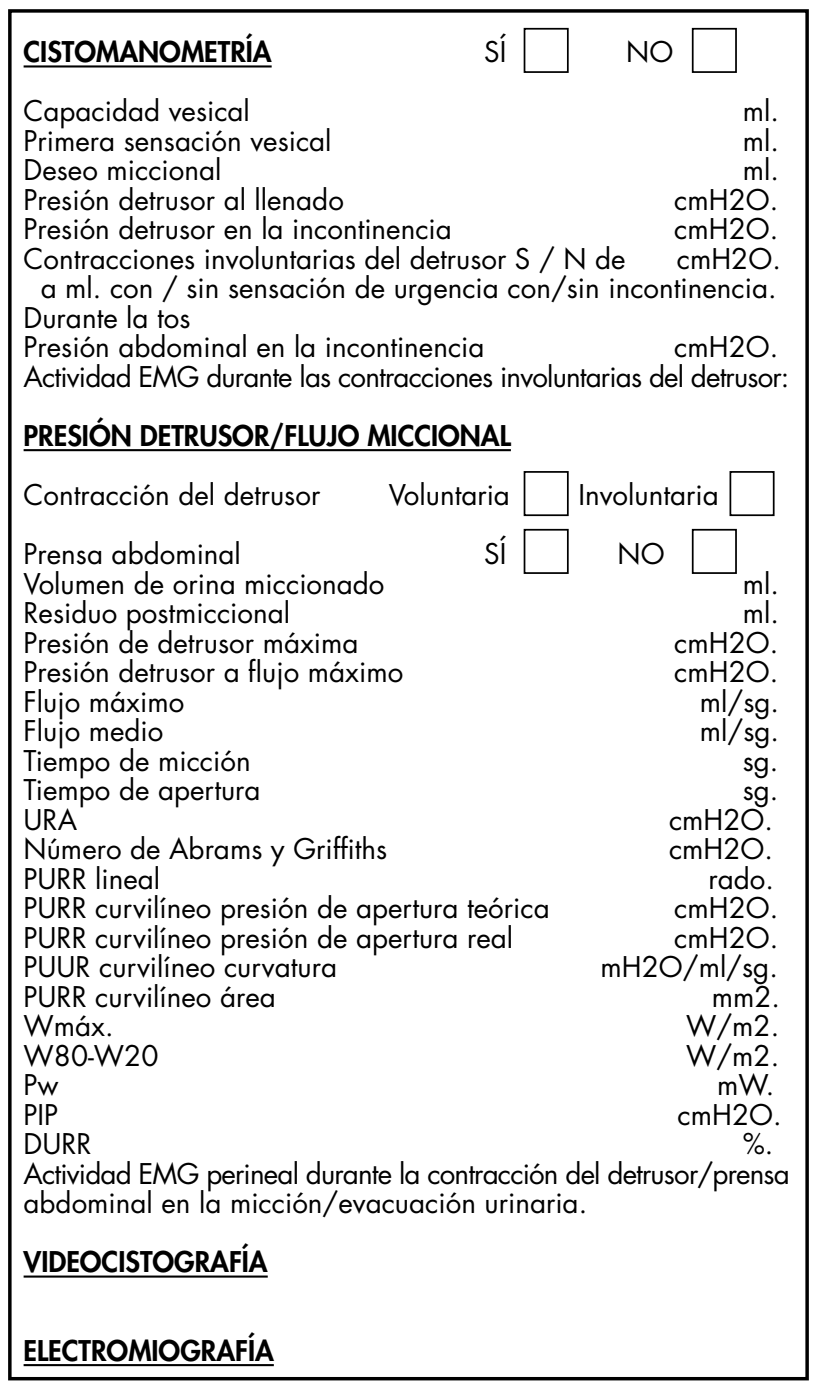

FIGURA IB. Protocolo del estudio urodinámico completo. Cistomanometría, presión detrusor/flujo miccional. pacientes evaluados antes y después de la cirugía, no siendo responsable de ningún caso de incontinencia postratamiento. Además, en el $87,5 \%$ de los casos desapareció tras la prostatectomía. Sí encontró datos preoperatorios interesantes entre los parámetros del perfil uretral (longitud uretral funcional media de 5,3 $+-7 \mathrm{~cm}$, presión máxima de cierre uretral en reposo de 78.7 +- 39.7 Cm H2O y voluntaria 137,7 +- 58,8 $\mathrm{cmH} 20)$.

Zermann Dh et al. (18) en el 2000 estudia 18 pacientes antes y después de la prostatectomía radical, incluyendo electromiografía con electrodos de aguja, pero excluyendo de esta electromiografía los pacientes que preoperatoriamente presentaron problemas en la fase de vaciado, no encontrando previo a la cirugía ningún paciente con síntomas irritativos y 6 pacientes con alteraciones del vaciamiento debidos a una aumento del tamaño prostático por HBP. En 11 pacientes en cambio encontró una control ineficiente voluntario del suelo pélvico, utilizando músculos auxiliares, pero sin hallazgos urodinámicos relevantes. Esto muestra una insuficiencia primaria de control central del suelo pélvico. En estos casos no encontró alteraciones ni en la aco-

DIAGNÓSTICO URODINÁMICO

\section{COMENTARIOS \\ SUGERENCIAS TERAPEUTICAS}

Médico responsable del estudio

Firma y fecha

FIGURA 1C. Protocolo del estudio urodinámico completo. Diagnóstico urodinámico, comentarios y sugerencias terapeuticas. 
modación, ni halló hiperactividad y la fluoroscopia fue normal.

John $\mathrm{H}$ et al. (19) en el 2000 en un estudio sobre 34 pacientes aporta como novedad el estudio de la transmisión de presión que fue determinada por la máxima presión uretral máxima dividida por la presión abdominal durante las maniobras de tos en una vejiga de volumen de $200 \mathrm{ml}$. y el umbral de sensibilidad uretral preoperatoriamente (umbral sensorial fue $16+/-11 \mathrm{~mA})$. Preoperatoriamente presentaron una presión máxima de cierre uretral de $49+/-10 \mathrm{~cm} \mathrm{H} 2 \mathrm{O}$, longitud media del esfínter de 50 +/- $7 \mathrm{~mm}$; Acomodación media de 49 +/- 35 $\mathrm{ml} / \mathrm{cmH} 2 \mathrm{O}$, y capacidad vesical $483+/-168 \mathrm{ml}$.

Castille et al. (20) (2003) en un estudio sobre 229 pacientes, en los que excluye a los pacientes incontinentes antes de la cirugía, realiza un estudio urodinámico preoperatorio con los siguientes resultados: $39,7 \%$ normales, obstrucción infravesical 24 $\%$, detrusor hipocontractil 8,3\%, detrusor hiperactivo $15,3 \%$, hiperactividad asociada a obstrucción 5,7 $\%$, acomodación vesical disminuida $1,7 \%$.

Giannantoni et al. (21) (2004) añade como novedad la utilización de la presión de fuga como evidencia de insuficiencia esfinteriana intrínseca. El estudio incluye a 49 pacientes, de los cuales 7 informan de urgencia $\circ$ urgencia-incontinencia pero sin necesidad de utilizar dispositivos anti-incontinencia. En el $57,1 \%$ de los pacientes encontró obstrucción, hiperactividad del detrusor en un 55,1 \%; el $20 \%$ de los pacientes que presentaron obstrucción tenían alteración de la contractilidad del detrusor. De todos los pacientes, el 42,8\% tenían disminución de la contractilidad. La acomodación estaba alterada en el 20,4\% de los pacientes. Ningún paciente mostró insuficiencia esfinteriana en el estudio preoperatorio. Necesitaban hacer presión abdominal en la micción el $81,6 \%$ de los pacientes. La acomodación fue de $37,1+14,9$ $\mathrm{ml} / \mathrm{cmH} 2 \mathrm{O}$, la Pmax del detrusor de $62,4+/-23,8$ $\mathrm{cmH} 2 \mathrm{O}$ y el flujo mxo. De $8,6+/-3,3 \mathrm{ml} / \mathrm{s}$.

Majoros A et al. (22) en 2006 en un estudio con 63 pacientes, encontraron un 58,7\% de estudios urodinámicos normales. $3,2 \%$ de los pacientes tenían debilidad esfinteriana, hiperactividad del detrusor aislada en un 14,3\%, obstrucción sólo 9,5 $\%$, hipocontractilidad en un 3,2 \% y acomodación disminuida en un 1,6\%. Un 9,5\% de los pacientes tenían obstrucción asociada a hiperactividad.

Hay que tener en cuenta que todos estos estudios se han realizados en varones susceptibles de una prostatectomía radical, con lo que el estadiaje preoperatorio correspondía a enfermedad localiza- da. Lo que ocurre, urodinámicamente, en pacientes con cánceres avanzados no hemos podido hallarlo en la literatura. Únicamente Heidenreich A et al. (23) (2008) informa de una tasa de continencia del 83 al $96 \%$ en pacientes sometidos a prostatectomía radical de salvación en pacientes con recurrencias locales previamente radiados.

Según Azzouzi et al. (24) (2007) los estudios urodinámicos preoperatorios muestran que la alteración más frecuente es la obstrucción infravesical, asociada en ocasiones con la hiperactividad detrusoriana, aunque esto no parece jugar un papel principal en la incontinencia post-prostatectomía. Lo mismo parece ocurrir con las alteraciones de la contractilidad, también en relación con la obstrucción. Puede que esto tenga alguna relación con la recuperación de la continencia. La medida preoperatoria del punto de fuga y de la longitud funcional uretral no parece aportar información significativa. Sin embargo, la medida de la presión máxima de cierre, tanto en reposo como durante la contracción voluntaria del esfínter, puede identificar un grupo de pacientes con mayor riesgo de incontinencia con valores alrededor de $60 \mathrm{cmH} 2 \mathrm{O}$ en reposo y de $120 \mathrm{~cm} \mathrm{H} 2 \mathrm{O}$ durante la contracción voluntaria. De importancia son las condiciones en las que se lleva a cabo el estudio urodinámico, tanto la velocidad de infusión, como su temperatura, pueden influir en los resultados.

\section{Valoración del Cáncer de próstata post-tratamiento}

Como es bien sabido, los tratamientos más frecuentes para el cáncer de próstata son la prostatectomía radical, la radioterapia, bien externa o en forma de braquiterapia y los tratamientos de bloqueo hormonal (aparte la quimioterapia sistémica) en aquellos casos en los que nos encontremos con pacientes con cánceres avanzados o en los que su situación clínica desaconsejen los procedimientos con fines radicales o curativos.

Nos centraremos en la prostatectomía radical y la radioterapia, dado que se dispone de mayor información en la literatura.

\section{Prostatectomía radical}

Como nos recuerda Moore $\mathrm{Km}$ et al. (25), desde que el Dr. Hugh Hampton Young en 1905 describiera la prostatectomía radical como tratamiento del cáncer de próstata, la incontinencia post-prostatectomía radical es una complicación de la intervención que afecta a la calidad de vida de los pacientes. Sus distintos aspectos se abordan en otros artículos 
de este número, pero es importante reseñar la dispersión de los datos. Podemos encontrar una incidencia desde un $8 \%$ de incontinencia total como nos indica Steiner MS et al. (26) en 1991, a un 90,9\% de continentes Hammerer et al. (16) en 1997, según las series consultadas (Tabla IV).

Krane RJ (27) (2000) refiere un intervalo del 0 al $57 \%$ de incontinencia post-prostatectomía según los distintos estudios. Si se refieren a la incontinencia total, no suele ser mayor a un $5 \%$. Además encuentra que la incontinencia aumenta con la edad, informando de un $10 \%$ de incontinencia total en los pacientes mayores de 70 años sometidos a prostatectomía radical. Propone como importante para la preservación de la continencia el músculo liso del mecanismo esfinteriano de la uretra distal, que se inicia en el suelo pélvico y termina en la unión uretro-prostática.

Sacco E et al. (28) (2006), revisando con un seguimiento de 24 meses a 985 hombres, encontraron una tasa de continencia de $83 \%, 92.3 \%$ y $93.4 \%$ dependiendo de la definición empleada de incontinencia:

1. No utilizan o sólo ocasionalmente material absorbente para la incontinencia.

2. Utilizan de 0 a 1 material absorbente diario pero con pérdidas ocasionales y

3. 0-1 material absorbente diarios.
Kundu SD et al. (29) (2004) del grupo de Catalona presenta una serie de 3477 prostatectomías radicales realizadas por él (el mismo cirujano) dando un $93 \%$ de recuperación de la continencia.

Es importante la realización de estudios urodinámicos en pacientes sometidos a prostatectomía radical ya que, como nos recuerda Foote J et al. (30) (1991), dada la prevalencia de disfunción vesical (es decir, alteración de la acomodación o hiperactividad del detrusor) como causa de incontinencia después de la prostatectomía, los estudios urodinámicos son esenciales en la evaluación de estos pacientes.

En este sentido también muestra su conformidad el trabajo de Azzouzzi AR et al. (2006) (24) en el que remarca la importancia del estudio urodinámico y la electromiografía. Considera que la evaluación urodinámica es el examen fundamental para cuantificar la presión de cierre uretral. Sienta las siguientes indicaciones:

- Incontinencia urinaria persistente a pesar de la rehabilitación perineal un año después de la cirugía.

- Antes en caso de incontinencia grave.

Salinas et al. (31) (1998) en un estudio sobre 25 pacientes, encuentra que el $68 \%$ de los pacientes recuperan la continencia total, siendo la tasa máxima de recuperación entre el tercer y cuarto mes

TABLA IV. PORCENTAJE DE PACIENTES CONTINENTES POST-PROSTATECTOMÍA.

\begin{tabular}{|c|c|c|}
\hline Autor & № pacientes & Continentes \\
\hline Steiner (1991) & 593 & $92 \%$ \\
\hline Hemmerer (1997) & 82 & $90.3 \%$ \\
\hline Salinas (1998) & 25 & $68 \%$ \\
\hline Kleinhans (1999) & 44 & $97,7 \%$ \\
\hline Golomb (1999) & 20 & $55 \%$ \\
\hline John (2000) & 34 & $82 \%$ \\
\hline Catalona (2004) & 1477 & $93 \%$ \\
\hline Sacco (2006) & 985 & $83 \%$ \\
\hline Majaros (2006) & 63 & $84,1 \%$ \\
\hline
\end{tabular}


tras la intervención. Demostraron que todos los pacientes, mediante video-cistografía, presentaban incompetencia del mecanismo de continencia proximal y que todos los pacientes incontinentes presentaban incontinencia urinaria de esfuerzo. Además, el 80 $\%$ de los pacientes incontinentes presentaban en la electromiografía potenciales de denervación del esfínter periuretral, no encontrándolos en ninguno de los pacientes continentes. Encontraron potenciales de reinervación en los pacientes continentes en un $50 \%$ de los casos, mientras que sólo se obtuvieron en un $20 \%$ de los incontinentes, siendo este dato estadísticamente significativo.

En el estudio de Ficazzola y Nitti VW (32) (1998) incluyen a 60 pacientes con estudio videourodinámico 6 meses después de la prostatectomía radical. La alteración intrínseca del esfínter se demostró en 54 pacientes (90\%). Alguna disfunción vesical se observó en 27 pacientes (45\%), incluída la hiperactividad del detrusor en 24 y disminución de la acomodación en 3. Las causas de la incontinencia fueron:

- Disfunción vesical 16 pacientes (27\%).

- Alteración intrínseca del esfínter aislada en 40 pacientes (67\%)

- Alteración intrínseca del esfínter y disfunción vesical en $14(23 \%)$

- Disfunción vesical aislada sólo 2 (3\%).

La incontinencia no se demostró en el estudio vídeo-urodinámico en 4 pacientes (7\%). De los 57 hombres que se quejaron de incontinencia urinaria de esfuerzo, en 54 se demostró alteración intrínseca del esfínter con un valor predictivo positivo de $95 \%$. En los 3 pacientes sin incontinencia urinaria de esfuerzo no se ha demostrado alteración intrínseca del esfínter en ninguno, con un valor predictivo negativo del $100 \%$. Los valores predictivos positivo y negativo de la incontinencia de urgencia fueron 44 y $81 \%$, respectivamente. Los autores concluyen que la incontinencia después de la prostatectomía radical está asociada con la alteración intrínseca del esfínter en la inmensa mayoría de los pacientes. La disfunción vesical rara vez es una causa aislada. No siempre que se demuestre en las pruebas urodinámicas una disfunción vesical es un factor importante en la incontinencia. El síntoma de incontinencia urinaria de esfuerzo (o su ausencia) predice con exactitud la presencia o ausencia de la alteración intrínseca del esfínter demostrable en el estudio urodinámico. No ocurre lo mismo con el síntoma urgencia-incontinencia, no siendo tan fiable, debido a la posible disfunción vesical.
Gudziak MR et al. (33) (1996) en un estudio retrospectivo sobre 27 pacientes, encontraron que el mecanismo responsable de la incontinencia postprostatectomía es la alteración intrínseca del esfínter, no estando relacionada con la máxima presión uretral (alteración extrínseca del esfínter).

Chao R y Mayo ME (34) (1995) en su estudio realizaron videurodinamias a 71 hombres con una media de 3,8 años tras la prostatectomía radical en los que persistía la incontinencia. Un total de 42 hombres (57\%) tenían alteración del esfínter aislada, $29(39 \%)$ presentaron hiperactividad del detrusor y / o disminución de la acomodación vesical en combinación con alteración esfinteriana y sólo $3(4 \%)$ presentaron hiperactividad del detrusor de forma aislada. De 29 hombres con una combinación de factores disminuyó la acomodación en 8 , y 3 también tenían hiperactividad del detrusor. La estenosis anastomótica ocurrió en el $24 \%$ de los pacientes. 31 pacientes vaciaban su vejiga con esfuerzo abdominal, sin contracción del detrusor demostrable (42\% general). Concluyen que la disminución de la acomodación es un hallazgo infrecuente después de la prostatectomía radical. Las anomalías del detrusor rara vez son la única causa de incontinencia, siendo la alteración del esfínter la que está presente en la mayoría de los pacientes.

Giannantoni et al. (35) (2008) realiza un estudio prospectivo con un seguimiento postoperatorio de 3 años completo de 38 pacientes. Hubo un aumento significativo en el número de pacientes con reducción de la acomodación vesical a los 8 meses de seguimiento. La reducción de la acomodación de nueva aparición se observó en el $32,3 \%$ de los pacientes y persiste en un $28,1 \%$ a los 36 meses de seguimiento. La hipocontractilidad del detrusor de nueva aparición se diagnosticó en el $51 \%$ de los pacientes a los 8 meses $(p<0,05)$ y persistió en el $25 \%$ de los casos 3 años después. Ningún paciente mostró residuo postmiccional. Las asociaciones entre la hipocontractilidad del detusor con hiperactividad detrusoriana y entre hipocontractilidad con la alteración intrínseca del esfínter se diagnosticó en $76,2 \%$ y el $44 \%$ de los pacientes, respectivamente, a los 8 meses, y en el $25 \%$ y en el $34 \%$ de los casos, respectivamente, a las 36 meses de seguimiento $(p<0,05$ у $p<0,001$ ). Los autores concluyen que estos datos reflejan probablemente una disfunción vesical secundaria a denervación durante la cirugía. Sin embargo, no producen síntomas miccionales, porque los pacientes desarrollan nuevas conductas miccionales.

Bentzon DN et al. (36) (2009) encuentran en su trabajo sobre 65 pacientes, que el estudio urodinámico postoperatorio después de 6 meses puede 
ser predictivo de la persistencia de la incontinencia, tanto de causa vesical como esfinteriana, sugiriendo una intervención más precoz. Estos autores diagnostican incontinencia de esfuerzo, encontrando una máxima presión de cierre uretral por debajo de 30 $\mathrm{cmH} 2 \mathrm{O}$, una disminución de la longitud funcional de la uretral y también un perfil distintivo.

John $\mathrm{H}$ et al. (37) (2000) han querido emplear el estudio urodinámico para tratar de esclarecer los mecanismos patofisiológicos de la incontinencia post-prostatectomía desde otro punto de vista. Estudian la sensibilidad de la uretra posterior y la transmisión de presiones, que son afectadas inmediatamente después de la prostatectomía. Una mejoría de estos parámetros después de 6 meses se asocia con la recuperación de la continencia. Estas observaciones sugieren que la continencia urinaria después de la prostatectomía radical depende de la integridad de la uretra posterior, la sensibilidad uretral y la eficacia de la transmisión de las presiones.

Respecto a la contractilidad del detrusor, Ishida $Y$ et al. (38) (2008) encontró de 80 pacientes sometidos a prostatectomía radical laparoscópica, que $6(7,5 \%)$ presentaba hipoconctactilidad del detrusor, que no estaba presente en el estudio preoperatorio.

En este sentido, Matsukawa $Y$ et al. (39) (2009) comparan la repercusión de la prostatectomía radical realizada por laparoscopia o abierta sobre la función vesical y uretral. La prostatectomía laparoscópica muestra una alteración de la fase de almacenamiento al disminuir la función del esfínter uretral y alterar la acomodación vesical. Estos autores no han encontrado diferencia significativa entre la cirugía laparoscópica y la abierta sobre la función uretral. Sin embargo, sí encontraron que los pacientes sometidos a prostatectomía abierta, tenían una menor acomodación vesical y un mayor porcentaje de hiperactividad del detrusor. Comparando pacientes incontinentes con continentes, encontraron diferencias entre ambos, presentando los incontinentes una disminución de la presión máxima de cierre uretral, una longitud funcional uretral disminuida, una menor acomodación y una mayor incidencia de hiperactividad del detrusor.

Presti JC Jr et al. (40) (1990) estudió distintos parámetros urodinámicos en pacientes tanto en 13 continentes como en 24 incontinentes tras la prostatectomía radical. Encontró una diferencia estadísticamente significativa entre los pacientes con y sin incontinencia para la media de la longitud funcional $(2,1$ frente a $3,6 \mathrm{~cm}$., Respectivamente, $p<0,001)$, la máxima presión de cierre uretral $(39$ frente a 74 $\mathrm{cmH} 2 \mathrm{O}$, respectivamente, $\mathrm{p}<0,001)$ y la máxima presión de cierre uretral durante la contracción voluntaria del esfínter externo $(107$ frente a $172 \mathrm{cmH} 2 \mathrm{O}$, respectivamente, $p<0,002)$. En este trabajo también encontraron que la tubularización por encima del nivel del esfínter externo estuvo presente en el continente, pero ausente en los pacientes con incontinencia. La continencia después de la prostatectomía radical depende de la eficiencia esfinteriana, que puede verse influida por la configuración anatómica del tracto de salida reconstruido y de la integridad del mecanismo esfinteriano uretral distal.

También se ha querido emplear el estudio urodinámico como predictor para el éxito o el fracaso del esfínter artificial como tratamiento en la incontinencia post-prostatectomía. Según Thiel DD et al. (41) (2007) no encuentran ningún parámetro que sea un predictor de fracaso del esfínter artificial, ni la hiperactividad del detrusor, ni la disminución en la primera sensación, ni la alteración de la acomodación o una baja capacidad vesical, según estos autores, son signos de mal pronóstico para la implantación de un esfínter artificial. Gomha MA et al. (42) (2003) tampoco encuentran parámetros demográficos ni urodinámicos que sean predictores de riesgo para los resultados del implante de un esfínter artificial entre aquellos pacientes que tienen patrones de vaciamiento distinto tras la prostatectomía radical (por contracción del detrusor o por presión abdominal).

En la patofisiología post-prostatectomía tiene también un lugar destacado la contractilidad del detrusor. En un estudio realizado por Natsume et al. (43) (2004) con tan sólo 17 pacientes, encontraron que los pacientes que tenían incontinencia presentaron una afectación de la contractilidad del detrusor, lo que se tradujo en un menor flujo, pero un aumento de la calidad de vida. La acomodación disminuida se recuperó. Estos autores no encuentran que ni la hiperactividad el detrusor ni el tratamiento antiandrogénico estén relacionados con la incontinencia prolongada. Aconsejan la evalulación urodinámica con atención al comportamiento del detrusor, para realizar un correcto manejo de cada paciente (Tabla V).

En 1999, Golomb J et al. (44) comparando los hallazgos urodinámicos pre y postoperatorios, encuentran una hiperactividad del detrusor en un $60 \%$ de los pacientes. En el post-operatorio el $55 \%$ son continentes, el $20 \%$ tiene incontinencia moderada de stress y el $25 \%$ episodios de urgencia-incontinencia. El valor predictivo positivo para la urgencia-incontinencia entre los pacientes con hiperactividad preoperatorio fue tan solo del $41,6 \%$. Concluyen que hay una correlación débil entre la hiperactividad preoperatoria y la urgencia incontinencia postoperatoria. 
En el trabajo ya citado de Castille $Y$ et al. (20) (2003) concluyen que ninguno de los pacientes diagnosticados de hiperactividad del detrusor y obstrucción vesical fue continente a las seis semanas siguientes a la cirugía. A los cuatro meses, a pesar de que mejora la continencia, los resultados siguen siendo significativamente más pobres que los observados en todos los demás grupos. Al contrario que en otros estudios encuentran que la evaluación urodinámica preoperatoria de pacientes programados para prostatectomía radical, permite identificar pacientes con alto riesgo de incontinencia urinaria postoperatoria.

Hellström et al. (1989) (14) en una serie de 19 pacientes encuentra un descenso significativo de la acomodación vesical y un incremento de las presiones vesicales e intrabdominales al flujo máximo, lo que interpreta como una descentralización parcial de la vejiga. El residuo postmiccional se reduce en el postoperatorio. Dos pacientes que permanecen totalmente incontinentes $(10,5 \%)$, presentaban presiones uretrales de cierre muy bajas.
Hammerer P y Huland (1997) (16) en el trabajo arriba expuesto, encontraron diferencias significativas entre la longitud funcional uretral $(27,6$ vs $20,5 \mathrm{~mm}$ ) y la presión máxima de cierre uretral $(68,1$ vs $53,1 \mathrm{~cm} \mathrm{H} 2 \mathrm{O}$ ) entre los pacientes incontinentes y continentes inmediatamente después de la intervención. A los 6 meses después de la prostatectomía hay un aumento tanto de la longitud funcional uretral como de la máxima presión de cierre, sin cambios respecto a los parámetros vesicales. Concluyen que los parámetros uretrales urodinámicos y la estabilidad del detrusor son factores importantes respecto a la continencia postquirúrgica.

Aboseif SR et al. (15) (1994), en un trabajo donde realizan estudios urodinámicos pre y postoperatorios al año de la prostatectomía, encuentran que, en el grupo de pacientes sin alteraciones urodinámicas previas a la intervención, la incontinencia es menos frecuente que en aquellos en los que sí encontraron alteraciones urodinámicas (3\% vs $39 \%)$. Es más, dependiendo del tipo de alteración, la frecuencia de incontinencia era distinta. Así, en aquellos pacien-

TABLA V. HALLAZGOS URODINÁMICOS POST-PROSTATECTOMIIA.

\begin{tabular}{|c|c|c|c|c|c|}
\hline Autor & № pacientes & $\begin{array}{c}\text { Hiperactividad } \\
\text { detrusor }\end{array}$ & $\begin{array}{l}\text { Acomodación } \\
\text { disminuida }\end{array}$ & $\begin{array}{l}\text { Afectación } \\
\text { contractilidad }\end{array}$ & Obstrucción \\
\hline Presti (1990) & 24 & $25 \%$ & & - & - \\
\hline Huckabay (1995) & 60 & $40 \%$ & $1,66 \%$ & - & $13 \%$ \\
\hline Chao (1995) & 71 & $39 \%$ & - & $42 \%$ & - \\
\hline Hammerer (1997) & 82 & $41 \%$ & - & - & - \\
\hline Salinas (1998) & 25 & $29 \%$ & - & - & $57 \%$ \\
\hline Ficazzola (1998) & 60 & $40 \%$ & $5 \%$ & - & - \\
\hline Majoros (2006) & 63 & $\begin{array}{l}30,2 \% \\
15,87 \% \\
\text { (de novo) }\end{array}$ & $1,58 \%$ & $3,17 \%$ & $\begin{array}{c}14,28 \% \\
12,7 \% \\
\text { (de novo) }\end{array}$ \\
\hline Gianantoni (2008) & 49 & $\begin{array}{c}10,2 \% \\
\text { (de novo) }\end{array}$ & $\begin{array}{c}10,2 \% \\
\text { (de novo) }\end{array}$ & $\begin{array}{c}10,2 \% \\
\text { (de novo) }\end{array}$ & $0 \%$ \\
\hline Ishida (2008) & 80 & - & - & $7.5 \%$ & - \\
\hline
\end{tabular}


tes que, preoperatoriamente tenían hiperactividad del detrusor presentarían el porcentaje menor con un $17 \%$, siendo el más alto $(71 \%)$ en aquellos pacientes que presentaban hiperactividad del detrusor y del esfínter. Concluyen por tanto que es útil realizar el estudio urodinámico para identificar el riesgo de incontinencia previa a la cirugía.

Marotta J et al. (45) (1995) comenta en su trabajo que no sólo habría que tomar en cuenta al esfínter como único protagonista de la incontinencia pos-prostatectomía, sino que, basado en sus estudios urodinámicos, debería tenerse en cuenta también la función uretral y el estado de la vejiga preoperatoriamente. El estudio urodinámico postoperatorio lo dejaría para aclarar las causas de la incontinencia.

En una revisión de la literatura llevada cabo por Porena M et al. (46) (2007), encontraron que el la disfunción del detrusor rara vez se presenta aislado, sino junto a la alteración intrínseca del esfínter. Los datos sobre la sensación de llenado vesical, capacidad en la cistomanometría, hiperactividad del detrusor, la afectación de la contractilidad del detrusor, la obstrucción del tracto de salida de la vejiga, son limitados y contradictorios. La hiperactividad del detrusor de novo es una disfunción en el $2 \%-77 \%$ de los pacientes. La alteración de la acomodación de la vejiga estuvo presente en el $8 \%-39 \%$ de los pacientes y fue de novo en torno al $50 \%$. La alteración de la contractilidad del detrusor se encontró en $29 \%$ $-61 \%$ de los pacientes, siendo de nueva aparición en un $47 \%$, y se recuperó en aproximadamente un $50 \%$ de los pacientes. El papel de estas disfunciones como agentes etiológicos de la incontinencia urinaria o de los síntomas de vaciado se evaluó de forma desigual. Estos autores proponen como conclusiones que la descentralización de la vejiga que se produce en el postoperatorio, la inflamación y / o infección, y la modificación geométrica de la pared vesical preexistentes, asociadas con hipoxemia con / sin fenómenos de neuroplasticidad, se han postulado como causas de la disfunción del detrusor. Sin embargo, la falta de estudios urodinámicos en el preoperatorio hace difícil evaluar las causas exactas de estas disfunciones. Por lo tanto, la urodinámica realizada, al menos en determinados casos, preoperatoriamente y durante el seguimiento puede ayudar a llegar a un diagnóstico preciso de las disfunciones, indicar el tratamiento adecuado y prevenir la incidencia y la aparición de la incontinencia urinaria postoperatoria.

Respecto al tiempo necesario para esperar la recuperación de la continencia, se ha establecido, como hemos visto, el estándar de un año, aunque en un reciente estudio realizado por Glickman $L$ et al. (47) (2009), con un seguimiento a largo plazo, han encontrado una mejoría en algún grado hasta un 23,4 $\%$ de los pacientes entre los 24 y 48 meses y una mejoría moderada a marcada de hasta un $12,2 \%$.

En el trabajo de Majoros A et al. (2006) (22) con 63 pacientes y un seguimiento de 9 meses postoperatorio, encontraron que en cuarenta y tres $(68,2 \%)$ y $53(84,1 \%)$ de los pacientes recuperaron la continencia a los 2 y 9 meses después de la intervención, respectivamente. Diez pacientes (15,9\%) fueron continentes inmediatamente después de la retirada del catéter. La incontinencia de esfuerzo urodinámica se detectó en $18(28,6 \%)$, hiperactividad del detrusor e incontinencia en $2(3,2 \%)$ pacientes 2 meses después de la cirugía. Las contracciones voluntarias esfinterianas preoperatorias fueron significativamente mayores en el grupo continente $(125$ frente a $96,5 \mathrm{cmH} 2 \mathrm{O}, \mathrm{P}$ $<0,0001)$. Los pacientes que fueron inmediatamente continentes tras la retirada del catéter no tenían síntomas del tracto urinario inferior (STUI) ni alteraciones urodinámicas en el preoperatorio. También presentaban una máxima presión de cierre uretral mayor tanto en el pre como en el postoperatorio (en reposo y durante la contracción voluntaria del esfínter) que los que tardaron más en recuperar la continencia. Los autores concluyen que lo más probable es que la principal causa de incontinencia después de la prostatectomía sea la alteración esfinteriana.

Se han postulado, por tanto, múltiples mecanismos patofisológicos desencadenados por la prostatectomía radical que pueden llevar a la incontinencia urinaria:

- Lesión esfinteriana directa.

- Afectación vesical

- Alteración de la inervación

- Alteración del suelo pélvico

El estudio del suelo pélvico es un nuevo enfoque. Zermann DH et al. (48) (2000) encuentra que durante los 6 meses posteriores a la intervención, la incontinencia va desapareciendo, a la vez que se normalizan los patrones electromiográficos de activación. Esto puede ser debido a que la prostatectomía altera los finos mecanismos de control central de la función muscular. La intervención quirúrgica puede, debido al daño tisular inherente, producir discretos daños nerviosos, inflamación, dolor, etc. alterar la rama aferente del sistema nervioso, apareciendo una alteración de los músculos del suelo pélvico. La hiperactividad del detrusor o del esfínter pueden considerarse un signo de cambios en la inervación debido a la manipulación quirúrgica o a situaciones patoneurofisiológicas preexistentes. La incompetencia pura del esfínter, la mayoría de las veces se debe a lesión quirúrgica. El biofeedback puede tener un 
efecto modulador sobre el SNC. La plasticidad del SNC también puede verse implicado.

Salinas et al. (1998) (31) demostraron que la persistencia de la denervación aguda del esfínter por encima de los cuatro meses de la prostatectomía radical se asocia a una incontinencia urinaria definitiva, evitando la espera de los 12 meses estándares, para la realización de la cirugía anti-incontinencia.

Respecto a la diferencia entre prostatectomía abierta, laparoscópica o robótica, Martínez-Salamanca JI y Allona Almagro A (49) (2007) afirman que, aunque en los estudios que implican una sola institución no parece haber diferencias significativas en cuanto a los resultados funcionales, cuando comparan distintos estudios, se encuentran con dificultades debido a la falta de randomización, los diferentes métodos y escalas de medida utilizadas, las diferentes definiciones, etc.

Se ha propuesto un protocolo para los pacientes incontinentes después de una prostatectomía, que según Huckabay $C$ et al. (50) (2005) consta de un estudio videurodinámico con cistomanometría, medición de la presión abdominal de fuga, test presión detrusor/flujo, valoración de la obstrucción según los nomogramas de la Sociedad Internacional de Continencia, estudio fluoroscópico y flujometría libre. En su estudio realizado sobre 60 pacientes, encontraron como más significativo la hiperactividad del detrusor en un $40 \%$ y un $13 \%$ acompañado de incontinencia. El 13,3\% de los pacientes estaban obstruidos.

\section{RADIOTERAPIA}

\section{Braquiterapia:}

En un interesante estudio realizado por Blaivas JG et al. (51) (2006) sobre 47 hombres sometidos a braquiterapia como tratamiento del cáncer de próstata, comparando los síntomas que presentaban, frente a una población de hombres con síntomas del tracto urinario inferior, pero sin cáncer de próstata, encontraron que la patofisiología de los síntomas en la población sometida a braquiterapia difería de la de la población general con síntomas del tracto urinario inferior. Así, vieron que existía una diferencia estadísticamente significativa entre los dos grupos respecto a la hiperactividad del detrusor, siendo más frecuente en los pacientes con braquiterapia $185 \%$ vs $47 \%$ ). Las estenosis uretrales (uretra posterior y anterior) también son más frecuentes en los pacientes tratados.

Para Wehle M et al. (52) (2004) el hecho de que un paciente presente flujos libres menores de
$10 \mathrm{ml} / \mathrm{s}$ (siendo estadísticamente significativo) o residuos mayores de $100 \mathrm{ml}$, son los parámetros urodinámicos no invasivos que pueden predecir complicaciones tras la braquiterapia.

Kollmeier MA et al. (53) (2005) observan en su trabajo, que se presenta incontinencia urinaria en un $18 \%$ de los pacientes sometidos resección transuretral de próstata después de la braquiterapia. No se observaron correlaciones entre la dosis de radiación, volumen prostático preimplante o la terapia hormonal. Los pacientes que presentan síntomas obstructivos (más de 2 años) después de la implantación que requieren RTUP se encuentran en mayor riesgo de incontinencia.

Por su parte Kielb SJ y Clemens JQ (54) (2005) estudian 146 estudios videourodinámicos realizados a otros tantos hombres que presentaban incontinencia después de una prostatectomía radical realizada unos 4 años de media antes del estudio. La incontinencia urinaria de esfuerzo (IUE) se demostró en 139 hombres (95\%), con una media de la presión abdominal de fuga de $59 \mathrm{~cm}$ de $\mathrm{H} 2 \mathrm{O}$. Se encontró correlación entre la fuga de presión estática y la presión del perfil uretral $(r=0,46, P<0,0001)$. La media de la presión uretral en las mediciones con IUE fue significativamente más baja que en los que tenían incontinencia de esfuerzo que en aquellos que no la presentaban $(46,6$ frente a $69 \mathrm{~cm} \mathrm{H} 2 \mathrm{O}, \mathrm{P}=0,001)$. Un total de 34 pacientes tenían disminución de la acomodación o hiperactividad del detrusor, pero este fue el único hallazgo en sólo 3 . Una respuesta del detrusor hipocontráctil se observó en 49 pacientes, y 35 de estos presentaban micción con prensa abdominal. Pacientes con radioterapia previa $(n=24)$ tenían más probabilidades de tener la obstrucción vesical; los otros parámetros fueron similares a los de pacientes sin radioterapia. Estos autores concluyen que la incontinencia después de la prostatectomía radical es causada por la alteración intrínseca del esfínter en la gran mayoría de los pacientes. Las mediciones de la presión del perfil uretral se correlacionan con la gravedad de la IUE, medida por la presión abdominal de fuga. La obstrucción vesical puede coexistir con la incontinencia urinaria de esfuerzo en una porción significativa de los pacientes. Durante la micción, se puede encontrar una respuesta del detrusor hipocontráctil, pero la importancia clínica de este hallazgo no está clara.

\section{Radioterapia externa:}

Son escasos los trabajos que hayan realizado estudios sobre incontinencia y radioterapia externa, y menos aún estudios urodinámicos en pacientes sometidos a radioterapia. 
Van Cangh PJ et al. (55) (1998) estudió de manera randomizada, la incontinencia en pacientes sometidos a radioterapia externa como adyuvante a la cirugía en el cáncer de próstata localizado. Se radió a estos pacientes por distintos motivos (márgenes positivos, afectación capsular o de las vesículas seminales, etc.). No encontró diferencias significativas en los pacientes sometidos a radioterapia en lo concerniente a su situación de continencia.

Resultados similares encuentra Formenti SC et al. (56) (2000) con radioterapia a dosis de adyuvancia (45-54 Gy).

Existe la creencia de que la resección transuretral de próstata después de la radioterapia tiene mayor riesgo de provocar incontinencia, bien por existir una lesión preexistente debido a la radioterapia o por afectación del esfínter debida a la extensión del propio tumor o por la lesión debida a la resección. Patel H et al. (57) (1997) en una corta serie de 7 pacientes sometidos a resección transuretral por obstrucción no encontraron un mayor riesgo de incontinencia urinaria.

Liu $M$ et al. (58) (2005) estudió a 1192 pacientes tratados de cáncer de próstata con radioterapia externa y con un seguimiento de al menos 24 meses. El 2,9\% presentaban incontinencia pretratamiento, más frecuente entre los pacientes sometidos a RTU prostática. Estos pacientes se excluyeron del estudio, por lo que desconocemos su evolución. En 57 $(4,9 \%)$ presentaron incontinencia tras la radioterapia, pero ninguno de grado IV. La resección transuretral post-radioterapia en este estudio aumentó cinco veces la probabilidad de padecer incontinencia urinaria.

Como nos recuerda Sandhu AS et al. (59) (2000) el hecho de haber presentado toxicidad urinaria aguda tras la radioterapia conformacional y el haber sido sometido a una resección previa, incrementa el riesgo de padecer incontinencia.

Lamentablemente no tenemos estudios urodinámicos suficientes que nos aclaren los mecanismos de la incontinencia en este grupo de pacientes.

\section{Hormonoterapia:}

Kollmeier MA et al. (2005) (53) no encuentra diferencia en lo que refiere a incontinencia en los pacientes sometidos a resección transuretral tras braquiterapia en función de la dosis de hormonoterapia que reciben.

Por su parte, Natsume et al. (2004) (43) no encuentran relación entre el tratamiento hormonal y la incontinencia prolongada.

\section{Otras terapias:}

También está descrita por Watanabe T et al. (60) (1996) en la literatura la incontinencia total por destrucción del esfínter por crioterapia. En el reciente estudio de Ritch CR et al. (61) 2009, obtienen de cuestionarios de calidad de vida una baja incidencia de incontinencia en pacientes tratados con aparatos de tercera generación. En un metanálisis realizado por Shelley $M$ et al. (62) (2007) encontramos una incidencia de incontinencia del 1,3 al 19\%.

Una de las complicaciones más frecuentes de la termoterapia en el tratamiento del cáncer de próstata es la incontinencia (63). Aunque como asegura Ficarra $V$ et al. (64) (2006), al año el $93 \%$ de los pacientes están continentes.

En conclusión, como nos recuerda Montanari E et al. (65) (2001), desde los años 90 hasta la actualidad, el peso de los distintos factores etiológicos de la incontinencia post-prostatectomía han ido cambiando, desde adjudicar hasta un $90 \%$ de causas vesicales (hiperactividad del detrusor) sola o como cofactor, hasta la actualidad, en la que la mayoría de las incontinencias se atribuyen a alteración intrínseca del esfínter. Hay que tener en cuenta que los datos pueden estar sesgados por:

1) falta de consenso sobre la definición de la continencia y / o incontinencia tras prostatectomía radical retropúbica

2) Las diferentes técnicas quirúrgicas que se comparan: preservación de una o dos bandeletas, reconstrucción del cuello vesical...

3) Los pacientes con incontinencia urinaria preoperatoria se incluyen en la series y el estado preoperatorio de la continencia en otros muchos no se conoce.

4) Las diferentes fechas en el registro de la incontinencia.

5) Diferentes métodos de recopilación de datos.

Si bien parece que la urodinámica ha aportado muchos conocimientos en este campo y que tiene un papel importante en el diagnóstico y planteamiento terapéutico de los pacientes con incontinencia grave prolongada post-prostatectomía, aún queda mucho por hacer.

Por tanto, podemos concluir que, si bien se ha recorrido un largo camino desde los albores del tratamiento del cáncer de próstata, en los que la incontinencia ya aparecía como una de las complicaciones frecuentes y con repercusiones sobre la cali- 
dad de vida de los pacientes, y habiendo sometido a miles de pacientes a estudios urodinámicos, sobre todo a aquellos que han padecido una prostatectomía radical, no estamos todavía hoy en condiciones de responder con total seguridad a las siguientes preguntas:

- ¿A qué pacientes se deben realizar estudios urodinámicos pre-tratamiento?

- De todos los factores implicados en la incontinencia en estos casos, el más importante sin duda es el esfinteriano, pero ¿̇cuál es la verdadera aportación de los otros factores (vesicales, perineales, inervación local, etc.) en la incontinencia y por tanto su posible repercusión en las terapias que se deban adoptar?

- ¿Puede y debe utilizarse la urodinámica como predictor de incontinencia?

- ¿Qué cambios en la fisiología del tracto urinario inferior ocurren realmente en los pacientes sometidos a radioterapia?

Esperemos que en los próximos años estas respuestas y muchas más vengan de la mano de la valoración urodinámica en estos pacientes.

\section{BIBLIOGRAFÍA y LECTURAS RECOMENDADAS ( ${ }^{*}$ lectura de interés $y^{* *}$ lectura fundamental)}

1. Melchior H. Urologe A. [Disorders of bladder function in the elderly][Article in German] 1995; 34(4):329-33.

2. Goode PS, Burgio KL, Redden DT, Markland A, Richter HE, Sawyer P, et al. Population based study of incidence and predictors of urinary incontinence in black and white older adults. J Urol. 2008; 179(4):1449-53; discussion 1453-4. Epub 2008 Mar 4.

3. Markland AD, Goode PS, Burgio KL, Redden DT, Richter HE, Sawyer P, et al. Correlates of urinary, fecal, and dual incontinence in older African-American and white men and women. J Am Geriatr Soc. 2008; 56(2):285-90. (Epub 2007 Dec 7).

4. Thom D. Variation in estimates of urinary incontinence prevalence in the community: effects of differences in definition, population characteristics, and study type .J Am Geriatr Soc. 1998; 46(4):473-80.

5. Irwin DE, Milsom I, Hunskaar S, Reilly K, Kopp $\mathrm{Z}$, Herschorn S, et al. Population-based survey of urinary incontinence, overactive bladder, and other lower urinary tract symptoms in five coun- tries: results of the EPIC study. Eur Urol. 2006; 50(6):1306-14; discussion 1314-5. (Epub 2006 Oct 2).

6. Dall'era MA, Hosang N, Konety B, Cowan JE, Carroll PR. Sociodemographic predictors of prostate cancer risk category at diagnosis: unique patterns of significant and insignificant disease. J Urol. 2009; 181(4):1622-7; discussion 1627. (Epub 2009 Feb 23).

7. Hampel C, Wienhold D, Benken N, Eggersmann C, Thuroff JW. Definition of overactive bladder and epidemiology of urinary incontinence. Urology 1997;50(6A Suppl):4-14, discussion 15-7.

8. Han E, Black LK, Lavelle JP. Incontinence related to management of benign prostatic hypertrophy. Am J Geriatr Pharmacother. 2007; 5(4):324-34.

*9. Nehra A, Jacobson D, McGree M, et al. Prostate cancer and urinary incontinence. The journal of urology 2009 Vol. 181, No. 4, Supplement, 591

10. Tan TL. Urinary incontinence in older persons: a simple approach to a complex problem. Ann Acad Med Singapore. 2003; 32(6):731-9.

11. Blaivas JG, de la Rocha RE. Impaired urinary flow rate: the distinction between bladder outlet obstruction and poor detrusor contractility. AUA Congress.Atlanta, 1986.

12. Salinas J, Virseda M, Teba F. Urodinámica de la hiperplasia prostática benigna. In Salinas J,

Virseda M, Teba F, eds. Aplicaciones diagnósticas, pronósticas y terapéuticas. Madrid: Pub Santher, 2000.

*13. Rudy DC, Woodside JR, Crawford ED. Urodynamic evaluation of incontinence in patients undergoing modified Campbell radical retropubic prostatectomy: a prospective study. J Urol. 1984; 132(4):708-12.

*14. Hellström P, Lukkarinen O, Kontturi M. Urodynamics in radical retropubic prostatectomy. Scand $\mathbf{J}$ Urol Nephrol. 1989;23(1):21-4.

**15. Aboseif SR, Konety B, Schmidt RA, Goldfien SH, Tanagho EA, Narayan PA. Preoperative urodynamic evaluation: does it predict the degree of urinary continence after radical retropubic prostatectomy? Urol Int. 1994;53(2):68-73.

*16. Hammerer P, Huland H. Urodynamic evaluation of changes in urinary control after radical retropubic prostatectomy. J Urol. 1997; 157(1):233-6.

**17. Kleinhans B, Gerharz E, Melekos M, Weingärtner K, Kälble T, Riedmiller H. Changes of urodynamic findings after radical retropubic prostatectomy. Eur Urol. 1999;35(3):217-21; discussion 221-2.

*18. Zermann DH, Ishigooka M, Wunderlich H, Reichelt $\mathrm{O}$, Schubert A study of pelvic floor function pre- and postradical prostatectomy using clinical neurourological investigations, urodynamics and electromyography. J. Eur Urol. 2000; 37(1):72-8. 
*19. John H, Sullivan MP, Bangerter U, Hauri D, Yalla SV. Effect of radical prostatectomy on sensory threshold and pressure transmission. J Urol. 2000; 163(6):1761-6. Comment in: J Urol. 2001; 166(4):1402.

**20. Castille Y, Opsomer RJ, Tombal B, Van Cangh PJ. Contribution of the preoperative urodynamic findings in the determination of risks factors of urinary incontinence after radical retropubic prostatectomy. Article in French. Ann Readapt Med Phys. 2003; 46(2):79-83.

**21. Giannantoni A, Mearini E, Di Stasi SM, Mearini L, Bini V, Pizzirusso G, et al. Assessment of bladder and urethral sphincter function before and after radical retropubic prostatectomy. J Urol. 2004; 171(4):1563-6.

*22. Majoros A, Bach D, Keszthelyi A, Hamvas A, Romics I. Urinary incontinence and voiding dysfunction after radical retropubic prostatectomy (prospective urodynamic study). Neurourol Urodyn. 2006; 25(1):2-7.

23. Heidenreich A, Semrau R, Thüer D, Pfister D. Radical salvage prostatectomy: Treatment of local recurrence of prostate cancer after radiotherapy. Article in German. Urologe A. 2008; 47(11):1441-6.

*24. Azzouzi AR, Ballereau C, Desgranchamps F, Devonec M, Fourmarier M, Haillot $\mathrm{O}$, et al. Management of male urinary incontinence after radical prostatectomy (CTMH AFU 2006-1/5): incidence and role of urodynamic assessment and electromyography. Article in French. Prog Urol. 2008; 18(1):14-8. (Epub 2008 Mar 4).

25. Moore KN. A review of the anatomy of the male continence mechanism and the cause of urinary incontinence after prostatectomy. J Wound Ostomy Continence Nurs. 1999; 26(2):86-93.

26. Steiner MS, Morton RA, Walsh PC. Impact of anatomical radical prostatectomy on urinary continence. J Urol. 1991; 145(3):512-4; discussion 514-5.

27. Krane RJ. Urinary incontinence after treatment for localized prostate cancer. Mol Urol. 2000; 4(3):279-86; discussion 287.

28. Sacco E, Prayer-Galetti T, Pinto F, Fracalanza S, Betto G, Pagano F, et al. Urinary incontinence after radical prostatectomy: incidence by definition, risk factors and temporal trend in a large series with a long-term follow-up. BJU Int. 2006; 97(6):1234-41.

*29. Kundu SD, Roehl KA, Eggener SE, Antenor JA, Han M, Catalona WJ. Potency, continence and complications in 3,477 consecutive radical retropubic prostatectomies J Urol. 2004; 172(6 Pt 1):2227-31.

*30. Foote J, Yun S, Leach GE. Postprostatectomy incontinence. Pathophysiology, evaluation, and management. Urol Clin North Am. 1991; 18(2):229-41.
**31. Salinas Casado J, Virseda Chamorro M, Fernández Ajubita H, Ramírez Fernández JC, Fuentes Márquez I, Resel Estévez L. Urodynamics of urinary incontinence post radical prostatectomy. Article in Spanish. Arch Esp Urol. 1998; 51(8):801-10.

**32. Ficazzola MA, Nitti VW The etiology of post-radical prostatectomy incontinence and correlation of symptoms with urodynamic findings. J Urol. 1998; 160(4):1317-20.

*33. Gudziak MR, McGuire EJ, Gormley EA. Urodynamic assessment of urethral sphincter function in post-prostatectomy incontinence. J Urol. 1996; 156(3):1131-4; discussion 1134-5.

**34. Chao R, Mayo ME. Incontinence after radical prostatectomy: detrusor or sphincter causes.

J Urol. 1995; 154(1):16-8. Comment in: J Urol. 1995; 154(1):184-5.

**35. Giannantoni A, Mearini E, Zucchi A, Costantini E, Mearini L, Bini V, Pet al. Bladder and urethral sphincter function after radical retropubic prostatectomy: a prospective long-term study. Eur Urol. 2008; 54(3):657-64. (Epub 2007 Nov 5). Comment in: Eur Urol. 2008; 54(3):664.

*36. Bentzon DN, Graugaard-Jensen C, Borre M. Urethral pressure profile 6 months after radical prostatectomy may be diagnostic of sphincteric incontinence: Preliminary data after 12 months' follow-up. Scand J Urol Nephrol. 2009; 43(2):114-8.

*37. John H, Sullivan MP, Bangerter U, Hauri D, Yalla SV. Effect of radical prostatectomy on sensory threshold and pressure transmission. J Urol. 2000; 163(6):1761-6. Comment in: J Urol. 2001; 166(4):1402.

38. Ishida Y, Matsukawa Y, Komatsu T, Yoshikawa Y, Hattori R, Goton M [Detrusor underactivity following laparoscopic radical prostatectomy. Article in Japanese. Hinyokika Kiyo. 2008; 54(4):25760.

39. Matsukawa Y, Hattori R, Yoshikawa Y, Ono Y, Gotoh M. Laparoscopic versus open radical prostatectomy: urodynamic evaluation of vesicourethral function. Int J Urol. 2009; 16(4):393-6.

$* * 40$. Presti JC Jr, Schmidt RA, Narayan PA, Carroll PR, Tanagho EA. Pathophysiology of urinary incontinence after radical prostatectomy. J Urol. 1990; 143(5):975-8.

**41. Thiel DD, Young PR, Broderick GA, Heckman MG, Wehle MJ, Igel TC, Petrou SP. Do clinical or urodynamic parameters predict artificial urinary sphincter outcome in post-radical prostatectomy incontinence? Urology. 2007; 69(2):315-9.

*42. Gomha MA, Boone TB. Voiding patterns in patients with post-prostatectomy incontinence: urodynamic and demographic analysis. J Urol. 2003; 169(5):1766-9. 
*43. Natsume O, Kondo H, Cho M, Fujimoto K, Ozono $\mathrm{S}$, Hirao Y. The impact of radical prostatectomy on patient well-being: a prospective urodynamic study focused on detrusor function. Hinyokika Kiyo. 2004; 50(1):1-6.

**44. Golomb J, Dotan Z, Leibovitch I, Mor Y, Ramon J. Can preoperative urodynamic examination allow us to predict the risk of incontinence after radical prostatectomy? Article in French. Prog Urol. 1999; 9(2):288-91.

*45. Marotta J, Lavianne P, Marievoet C. Value of urodynamic studies in the framework of total radical prostatectomy. Article in French. Acta Urol Belg. 1995; 63(1):25-35.

**46. Porena M, Mearini E, Mearini L, Vianello A, Giannantoni A. Voiding dysfunction after radical retropubic prostatectomy: more than external urethral sphincter deficiency. Eur Urol. 2007 52(1):38-45. (Epub 2007 Mar 26). Comment in: Eur Urol. 2007; 52(1):1-2.

47. Glickman L, Godoy G, Lepor H. Changes in continence and erectile function between 2 and 4 years after radical prostatectomy. J Urol. 2009; 181(2):731-5. (Epub 2008 Dec 16).

*48. Zermann DH, Ishigooka M, Wunderlich H, Reichelt $\mathrm{O}$, Schubert A study of pelvic floor function pre- and postradical prostatectomy using clinical neurourological investigations, urodynamics and electromyography. J.Eur Urol. 2000; 37(1):72-8.

49. Martínez-Salamanca JI, Allona Almagro A. Radical prostatectomy: open, laparoscopic and robotic. Looking for a new gold standard?. Article in Spanish. Actas Urol Esp. 2007; 31(4):316-27.

*50. Huckabay C, Twiss C, Berger A, Nitti VW. A urodynamics protocol to optimally assess men with post-prostatectomy incontinence. Neurourol Urodyn. 2005; 24(7):622-6.

*51. Blaivas JG, Weiss JP, Jones M. The pathophysiology of lower urinary tract symptoms after brachytherapy for prostate cancer. BJU Int. 2006; 98(6):1233-7; discussion 1237.

52. Wehle MJ, Lisson SW, Buskirk SJ, Broderick GA, Young PR, Igel TC. Prediction of genitourinary tract morbidity after brachytherapy for prostate adenocarcinoma. Mayo Clin Proc. 2004; 79(3):314-7. Comment in: Mayo Clin Proc. 2004; 79(7):945-6; author reply 947-9. Mayo Clin Proc. 2004; 79(7):945; author reply 947-8. Mayo Clin Proc. 2004; 79(7):946-7; author reply 947-9. Mayo Clin Proc. 2004; 79(3):307-8.

53. Kollmeier MA, Stock RG, Cesaretti J, Stone NN. Urinary morbidity and incontinence following transurethral resection of the prostate after brachytherapy. J Urol. 2005; 173(3):808-12.

*54. Kielb SJ, Clemens JQ. Comprehensive urodynamics evaluation of 146 men with incontinen- ce after radical prostatectomy. Urology. 2005; 66(2):392-6.

55. Van Cangh PJ, Richard F, Lorge F, Castille Y, Moxhon A, Opsomer R, et al. Adjuvant radiation therapy does not cause urinary incontinence after radical prostatectomy: results of a prospective randomized study. J Urol. 1998; 159(1):164-6. Comment in: J Urol. 1998; 159(1):172.

56. Formenti SC, Lieskovsky G, Skinner D, Tsao-Wei DD, Groshen S, Petrovich Z. Update on impact of moderate dose of adjuvant radiation on urinary continence and sexual potency in prostate cancer patients treated with nerve-sparing prostatectomy. Urology. 2000; 56(3):453-8.

57. Patel H, Peddada AV, Zimmern PE, Hernandez R, Kagan R. Risk of incontinence with transurethral resection of the prostate after radiation therapy for prostate cancer J Surg Oncol. 1997; 64(2):127-9.

58. Liu M, Pickles T, Berthelet E, Agranovich A, Kwan W, Tyldesley S, McKenzie M, Keyes M, Morris J, Pai H; Prostate Cohort Initiative. Urinary incontinence in prostate cancer patients treated with external beam radiotherapy. Radiother Oncol. 2005; 74(2):197-201.

59. Sandhu AS, Zelefsky MJ, Lee HJ, Lombardi D, Fuks Z, Leibel SA. Long-term urinary toxicity after 3-dimensional conformal radiotherapy for prostate cancer in patients with prior history of transurethral resection. Int J Radiat Oncol Biol Phys. 2000; 48(3):643-7.

60. Watanabe T, Rivas DA, Miettinen M, Gomella L, Chancellor MB. Total incontinence secondary to sphincter destruction after prostate cryotherapy for recurrent prostatic carcinoma. Int $\mathbf{J}$ Urol. 1996; 3(4):324-5.

61. Ritch CR, Katz AE Update on cryotherapy for localized prostate cancer. Curr Urol Rep. 2009; 10(3):206-11.

62. Shelley M, Wilt TJ, Coles B, Mason MD. Cryotherapy for localised prostate cancer.Cochrane Database Syst Rev. 2007; (3):CD005010.

63. Rebillard X, Soulié M, Chartier-Kastler E, Davin JL, Mignard JP, Moreau JL, et al. Association Francaise d'Urologie. High-intensity focused ultrasound in prostate cancer; a systematic literature review of the French Association of Urology. BJU Int. 2008; 101(10):1205-13. Epub 2008 Mar 4.

64. Ficarra V, Antoniolli SZ, Novara G, Parisi A, Fracalanza S, Martignoni G, et al. Short-term outcome after high-intensity focused ultrasound in the treatment of patients with high-risk prostate cancer. BJU Int. 2006; 98(6):1193-8.

65. Montanari E, Del Nero A, Bernardini P, Trinchieri A, Zanetti G, Rocco B. Epidemiology and physiopathology of urinary incontinence after radical prostatectomy. Article in Italian. Arch Ital Urol Androl. 2001; 73(3):121-6. 